\title{
Avaliação do uso de antimicrobianos em idosos internados em um hospital público
}

\author{
Evaluation of antimicrobial use in elderly hospitalized in a public hospital \\ Evaluación del uso de antimicrobianos em mayores hospitalizados em um hospital público
}

Recebido: 16/09/2021 | Revisado: 26/09/2021 | Aceito: 09/11/2021 | Publicado: 14/11/2021

\author{
Monique Gomes Cerqueira \\ ORCID: https://orcid.org/0000-0003-3098-3389 \\ Faculdade de Tecnologia e Ciências, Brasil \\ E-mail: moniquegoomes42@gmail.com \\ Rafaela Lima Santos \\ ORCID: https://orcid.org/0000-0001-7779-4556 \\ Universidade Estadual do Sudoeste da Bahia, Brasil \\ E-mail: rafa_guapa@hotmail.com. \\ Priscilla Magalhães Souza \\ ORCID: https://orcid.org/0000-0002-4764-7034 \\ Faculdade de Tecnologia e Ciências, Brasil \\ E-mail: priscillamagalhes86@gmail.com \\ Gildomar Lima Valasques Junior \\ ORCID: https://orcid.org/0000-0002-2877-5313 \\ Universidade Estadual do Sudoeste da Bahia, Brasil \\ E-mail: gildomar.valasques@uesb.edu.br \\ Tuany Santos Souza \\ ORCID: https://orcid.org/0000-0003-0165-4201 \\ Universidade Estadual do Sudoeste da Bahia, Brasil \\ Faculdade de Tecnologia e Ciências, Brasil \\ E-mail: tssouza.jeq@ftc.edu.br
}

\begin{abstract}
Resumo
Objetivo: O objetivo deste estudo foi avaliar o uso de antimicrobianos em idosos internados em um hospital público. Metodologia: Trata-se de um estudo com delineamento transversal, de caráter quantitativo e descritivo, realizado na Clínica Médica de um hospital público da Bahia, durante um período de três meses, através de um formulário estruturado pelo pesquisador. Foram analisados 42 prontuários de pacientes com idade igual ou acima de 60 anos. Destes, 29 faziam uso de antimicrobianos. Resultados: A prevalência do uso de ATMs foi de (69\%), sendo maior no sexo feminino (93\%), e nos pacientes com 80 anos ou mais (52\%). Os medicamentos mais utilizados foram a ceftriaxona (59\%), levofloxacino (28\%) e azitromicina (28\%). Houve predomínio da polifarmácia (100\%). Além disso, os principais diagnósticos para o uso dos ATMs foram para as doenças que acometem o aparelho respiratório (38\%) e doenças do aparelho circulatório (32\%). Em relação a via de administração, foi observado a maior frequência no uso da via intravenosa (79\%). Conclusão: Sugere-se que é necessário conhecer o uso desses medicamentos nos hospitais, principalmente na geriatria, para subsidiar a elaboração de protocolos clínicos, reduzir o tratamento por terapia empírica, além de ser importante a interação da equipe multiprofissional para evitar o aumento da resistência bacteriana e impedir que possíveis quanto as prescrições ocorram.
\end{abstract}

Palavras-chave: Anti-infecciosos; Idoso; Hospitalização.

\begin{abstract}
Objective: The aim of this study was to evaluate the use of antimicrobials in elderly people admitted to a public hospital. Methodology: This is a cross-sectional study, quantitative and descriptive, carried out in the Medical Clinic of a public hospital in Bahia, over a period of three months, using a form structured by the researcher. Forty-two medical records of patients aged over 60 years were analyzed. Of these, 29 were using antimicrobials. Results: The prevalence of TMJ use was (69\%), being higher in females (93\%) and in patients aged 80 years or older (52\%). The most used drugs were ceftriaxone (59\%), levofloxacin (28\%) and azithromycin (28\%). There was a predominance of polypharmacy (100\%). In addition, the main diagnoses for the use of TMJs were for diseases that affect the respiratory system (38\%) and diseases of the circulatory system (32\%). Regarding the route of administration, the highest frequency of use of the intravenous route was observed (79\%). Conclusion: It is suggested that it is necessary to know the use of these drugs in hospitals, especially in geriatrics, to support the development of clinical protocols, reduce treatment by empirical therapy, in addition to the importance of the interaction of the multidisciplinary team to avoid the increase in resistance bacterial and prevent as much as possible prescriptions from occurring.
\end{abstract}

Keywords: Anti-infectives; Old man; Hospitalization. 


\begin{abstract}
Resumen
Objetivo: El objetivo de este estudio fue evaluar el uso de antimicrobianos en ancianos ingresados en un hospital público. Metodología: Se trata de un estudio transversal, cuantitativo y descriptivo, realizado en la Clínica Médica de un hospital público de Bahía, durante un período de tres meses, utilizando un formulario estructurado por el investigador. Se analizaron 42 historias clínicas de pacientes mayores de 60 años. De estos, 29 estaban usando antimicrobianos. Resultados: La prevalencia de uso de ATM fue (69\%), siendo mayor en mujeres (93\%) y en pacientes de 80 años o más (52\%). Los fármacos más utilizados fueron ceftriaxona (59\%), levofloxacina (28\%) y azitromicina (28\%). Predominó la polifarmacia (100\%). Además, los principales diagnósticos para el uso de ATM fueron para enfermedades que afectan el sistema respiratorio (38\%) y enfermedades del sistema circulatorio (32\%). En cuanto a la vía de administración, se observó la mayor frecuencia de uso de la vía intravenosa (79\%). Conclusión: Se sugiere que es necesario conocer el uso de estos fármacos en hospitales, especialmente en geriatría, para apoyar el desarrollo de protocolos clínicos, reducir el tratamiento por terapia empírica, además de la importancia de la interacción del equipo multidisciplinario para evitar el aumento de la resistencia bacteriana y evitar en la medida de lo posible que se produzcan prescripciones.
\end{abstract}

Palabras clave: Antiinfecciosos; Anciano; Hospitalización.

\title{
1. Introdução
}

O crescimento da população idosa tem se intensificado em todo o mundo, o que causa um impacto principalmente nos sistemas de saúde. No Brasil em 2017, o número de idosos com 60 anos ou mais ultrapassou um percentual de 30,2 milhões e, com isso, entende-se que esse fenômeno está relacionado com a mudança de hábitos da sociedade e com a transição demográfica observada nos últimos anos (Flores et al., 2019).

Do ponto de vista epidemiológico, os idosos representam a faixa etária que mais frequentam os serviços de saúde devido ao desenvolvimento de doenças crônicas associado às mudanças fisiológicas do avançar da idade que culminam em um declínio progressivo e funcional, bem como cognitivo, psíquico e social (Goulart et al., 2014). Tais alterações fisiológicas estão geralmente associadas a polifarmácia, tornando-os mais expostos à utilização de medicamentos potencialmente inapropriados, a maior propensão a ocorrência de interações medicamentosas, bem como reações adversas aos medicamentos (RAM) (Rodrigues \& Oliveira, 2016).

Cabe ressaltar que as alterações morfofisiológicas do envelhecimento também afetam o sistema imunológico, tornando esses indivíduos mais suscetíveis ao surgimento de infecções e, por consequência, à necessidade de hospitalizações e do uso de antimicrobianos (Bertol et al., 2020; Izaías et al., 2014; Silva \& Garbaccio, 2016). Por essa razão, no ambiente hospitalar os antimicrobianos (ATM) correspondem à uma das classes de fármacos mais prescritas para idosos e, especificamente nessa população, merecem uma abordagem cuidadosa e específica por parte da equipe de saúde, tendo em vista que estes fármacos devem ser usados como indicação terapêutica de doenças infecciosas ou para fins profiláticos (Souza \& Silva, 2015).

Neste sentido, o uso inadequado dos ATM tem ocasionado o aparecimento de cepas bacterianas resistentes, o que tem sido uma preocupação mundial, por gerar aumento nas taxas de morbidade e mortalidade, além de intensificar danos individuais e custos aos sistemas de saúde (Dylis et al., 2019; Silva \& Garbaccio, 2016), devido à reações adversas que podem ocorrer como consequência de um tratamento não seguro e ineficaz (Cazarim \& Araújo, 2011; (Giarratano et al.,2018). Além disso, segundo Gleeson et al. (2020), a ocorrência de erros de medicações envolvendo os ATM contribui para o aumento do tempo de permanência hospitalar, podendo agravar ainda mais os eventos adversos, de modo a comprometer a segurança do paciente.

O uso desta classe de medicamentos é, de fato, um grande problema em muitos países, pois as prescrições hospitalares em alguns casos são inapropriadas, já que é evidente que os indivíduos mais velhos estejam frente a um maior risco de prescrições potencialmente inapropriadas (PIPs) de ATM, sejam elas relacionados ao tempo de tratamento, ou até mesmo ao tempo de internação do paciente, visto que, os conhecimentos são insuficientes para um diagnóstico conclusivo, devido às 
múltiplas comorbidades desses indivíduos o que, frequentemente, implica em uma falha terapêutica e no crescimento da resistência bacteriana (Tavares et al., 2015; Baclet et al., 2017).

Desta forma, considera-se indispensável a realização de uma análise prospectiva dos ATM utilizados no âmbito hospitalar, com a finalidade de avaliar as indicações do uso. Além disso, torna-se relevante, evidenciar a participação e acompanhamento multiprofissional aos idosos na adoção de medidas de precauções, principalmente no que compete a minimização de possíveis erros de medicação, desde a prescrição, dispensação e administração, levando-se em consideração a especificação da duração do tratamento, doses, posologia, dentre outras variáveis. Tais avaliações possibilitam a capacidade de compreender a qualidade da terapia e podem proporcionar a melhoria de condutas profissionais que refletirão em um tratamento eficaz e seguro para o paciente. Mediante a esses fatores, o presente estudo tem como objetivo avaliar o uso de antimicrobianos em idosos internados em um hospital público.

\section{Metodologia}

\subsection{Tipo de Estudo}

Este estudo possui delineamento transversal prospectivo, de caráter quantitativo, descritivo e retrospectivo (Pereira et al., 2018).

\subsection{Campo e Cenário da Pesquisa}

O campo da pesquisa foi o município de Jequié-Ba, que abrange uma população estimada em torno de 156.126 habitantes, uma área territorial de 2.969, $039 \mathrm{~km}^{2}$ sendo que referenciam para esta unidade 27 municípios. O cenário do estudo foi a clínica médica de um hospital público regional, que atende em regime porta aberta. A unidade possui 276 leitos para diversas especialidades, além da clínica médica, pediatria, neurologia, psiquiatria e terapia intensiva (SESAB, 2020).

\subsection{Sujeitos do estudo e critérios de elegibilidade}

Para realização do estudo foram incluídos os prontuários de pacientes idosos, de ambos os sexos, com idade igual ou superior à de 60 anos (WHO, 2002) internados na clínica médica. Ademais, esses pacientes estavam em uso de pelo menos um ATM por via oral, ou parenteral sistêmica, além de ter mais de vinte quatro horas de permanência no setor. Foram excluídas prescrições ilegíveis, prescrições de pacientes que durante o tempo de internação foram à óbito ou aquelas com ATM prescritos para uso tópico.

\subsection{Procedimentos de coleta e variáveis do estudo}

Os dados foram coletados nos prontuários a partir de um formulário estruturado pelo pesquisador contendo o número do registro do paciente, no período de novembro de 2018 a janeiro de 2019, conforme o Apêndice 1.

No que se refere as variáveis de estudo, estas estão classificados em dois subgrupos: dependentes e independentes. A variável dependente é o uso de antimicrobianos em idosos. Em relação as variáveis independentes, foram avaliados e coletados os seguintes dados sociodemográficos e clínicos: sexo, idade, motivo de internação, diagnóstico conclusivo avaliados de acordo a Classificação Internacional de Doenças (WHO, 2020) e período de internação.

Para a caracterização do uso de ATMs foram observadas as seguintes variáveis: Uso de ATM, dose, quantidade de ATM, via de administração, especialidade do prescritor e número de medicamentos prescritos. Foram avaliadas as classes farmacológicas predominantes dos ATM prescritos, e caracterizadas de acordo com a classificação Anatomical Therapeutic 
Chemical (ATC), no nível $3^{\circ}$ (WHO, 2018). Além disso, foram identificados a ocorrência da polifarmácia, de acordo aos critérios da OMS, como o uso concomitante de cinco ou mais medicamentos por paciente (WHO, 2002).

\subsection{Análise dos dados}

O programa utilizado para tabular os dados foi o Microsoft Office Excel® (versão 15.0) 2013 e, posteriormente, a análise descritiva foi realizada no software Statistical Package for the Social Sciences® (SPSS, 2012), versão 21.0. Foram realizadas as distribuições de frequências absolutas e relativas para as variáveis categóricas, bem como a média e o desvio padrão para as variáveis contínuas numéricas.

\subsection{Aspectos éticos}

O presente estudo está vinculado ao projeto de pesquisa "Seguimento farmacoterapêutico e reconciliação medicamentosa em pacientes internados no Hospital Geral Prado Valadares, localizado no município de Jequié" que corresponde aos princípios éticos da Resolução 466/12 (BRASIL, 2012) do Conselho Nacional de Saúde (CNS), e está aprovado pelo Comitê de Ética em Pesquisa (CEP) da Universidade Estadual do Sudoeste da Bahia (UESB) conforme o seguinte número do parecer: 462.333 e CAAE de número 21431313.5.0000.0055.

\section{Resultados}

Foram analisados 42 prontuários de pacientes com idade igual ou acima de 60 anos, referindo-se a todos os pacientes idosos que estiveram internados no setor da clínica médica do hospital durante o período de realização deste estudo. Observouse que o sexo feminino foi maioria entre os pacientes (93\%), a idade média dos mesmos foi dede 77,86 anos $( \pm 10,43)$, sendo a idade mínima 62 e a máxima 99 anos. Houve predominância entre os pacientes idosos com 80 anos ou mais, ou seja, os longevos, com um percentual de 44\% (n=18). Os demais fatores sociodemográficos e clínicos da amostra analisa descritos na Tabela 1.

No que se refere ao motivo de internação, foi observado que apenas 55\% (n=23) tinham informação no prontuário. Conforme o CID-11 esses motivos de internação configuraram-se como sinais, sintomas ou achados classificados em outra parte $(43 \% ; n=10)$, seguido de doenças do sistema circulatório $(39 \% ; n=9)$, doenças que acometem o sistema respiratório (30\%; $\mathrm{n}=7)$, dentre outros. Quanto aos diagnósticos conclusivos observou-se que $(60 \% ; \mathrm{n}=25)$, do total de prontuário verificados continham informações. E dentre estes diagnósticos, as doenças que acometem o sistema circulatório foram as mais prevalentes, com $60 \%(\mathrm{n}=15)$, seguido das doenças do sistema respiratório, com $36 \%(\mathrm{n}=9)$, doenças endócrinas nutricionais e metabólicas, que somaram $32 \%(\mathrm{n}=8)$.

No que diz respeito ao período de internação, a maior predominância foram os pacientes internados em um período de 7-14 dias (48\%; n=20), destacando-se maior período de internação em 42 dias e o menor de 7 dias, com uma média de 15 dias $( \pm 7,6)$. Entretanto, após o período da coleta, três pacientes permaneceram internados, não sendo possível mensurar as suas respectivas informações. Quanto ao peso e duração do tratamento em 100\% dos prontuários não havia informações sobre ambas as variáveis. 
Tabela 1- Fatores sociodemográficos e clínicos de pacientes idosos internados no setor da clínica médica de um hospital público. Bahia, Brasil, 2018-2019.

\begin{tabular}{|c|c|c|}
\hline Variáveis & $\mathbf{N}$ & $\%$ \\
\hline \multicolumn{3}{|l|}{$\operatorname{Sexo}(n=42)$} \\
\hline Feminino & 39 & 93 \\
\hline Masculino & 3 & 7 \\
\hline \multicolumn{3}{|l|}{ Idade $(n=42)$} \\
\hline $60-69$ & 13 & 32 \\
\hline $70-79$ & 11 & 26 \\
\hline 80 e mais & 18 & 44 \\
\hline \multicolumn{3}{|l|}{ Motivo de Internação* $(\mathbf{n}=\mathbf{2 3})$} \\
\hline Sinais, sintomas ou achados classificados em outra parte & 10 & 43 \\
\hline Doenças do aparelho circulatório & 9 & 39 \\
\hline Doenças do aparelho respiratório & 7 & 30 \\
\hline Lesões ou certas consequências de causas externas & 5 & 22 \\
\hline Algumas doenças infecciosas ou parasitárias & 1 & 4 \\
\hline Doenças endócrinas, nutricionais ou metabólicas & 1 & 4 \\
\hline Outros & 3 & 13 \\
\hline Não informado & 19 & 82 \\
\hline \multicolumn{3}{|l|}{ Diagnóstico Conclusivo* (CID-11) (n=25) } \\
\hline Doenças do aparelho circulatório & 15 & 60 \\
\hline Doenças do aparelho respiratório & 9 & 36 \\
\hline Doenças endócrinas, nutricionais ou metabólicas & 8 & 32 \\
\hline Doenças do aparelho digestivo & 3 & 12 \\
\hline Sinais, sintomas ou achados classificados em outra parte & 3 & 12 \\
\hline Algumas doenças infecciosas ou parasitárias & 3 & 12 \\
\hline Lesão, envenenamento ou certas consequências de causa externas & 1 & 4 \\
\hline Neoplasias & 1 & 4 \\
\hline Outros & 3 & 12 \\
\hline Não informado & 17 & 68 \\
\hline \multicolumn{3}{|l|}{ Período de internação** $(n=42)$} \\
\hline $7-14$ dias & 20 & 48 \\
\hline $15-21$ dias & 13 & 33 \\
\hline 22 ou mais dias & 6 & 19 \\
\hline
\end{tabular}

(*) Classificação internacional de doenças CID-11.

(**) Pacientes que continuaram internados: $(7 \% ; n=3)$. Fonte: Autores (2020).

A Tabela 2 mostra a caracterização do uso de ATMs pelos idosos deste estudo, onde constatou-se que a prevalência do uso de ATMs foi de 69\% ( $\mathrm{n}=29$ ), sendo maior no sexo feminino (93\%), e nos pacientes com 80 anos ou mais (52\%). No que concerne ao motivo de internação, a predominância foi para as doenças do aparelho respiratório (28\%; $\mathrm{n}=5$ ), seguida por Lesão, envenenamento ou certas consequências de causas externas (28\%; n=5). Enquanto que para os diagnósticos, a prevalência foi para doenças do aparelho respiratório (38\%), e doenças do aparelho circulatório (32\%). No que se refere a quantidade desses medicamentos por paciente, a maioria dos idosos estavam em uso de dois ATMs (52\%), sendo a média de 1,6 (desvio padrão 0,63 ) por paciente.

Quanto ao período de internação dos ATMs, a maior predominância foi para os pacientes internados de 7-14 dias (41\%; n=14), destacando-se o maior período em 42 dias e o menor 7 dias, com uma média de 15 dias (desvio padrão 7,6).

Em relação à via de administração, observa-se a maior frequência no uso da via intravenosa (79\%), seguida pela via oral $(41 \%)$ e $(3,4 \%)$ não constava nenhuma informação. Quanto a especialidade do prescritor, houve predomínio por um clínico geral $97 \%$. A prática da polifarmácia foi observada em $100 \%$ dos prontuários. 
Tabela 2 - Caracterização do uso de antimicrobianos (ATMs) de pacientes idosos internados no setor da clínica médica de um hospital público. Bahia, Brasil, 2018-2019.

\begin{tabular}{|c|c|c|c|}
\hline Variáveis & $\begin{array}{c}\text { Taxa } \\
\text { Resposta }\end{array}$ & $\mathbf{N}$ & $\%$ \\
\hline \multicolumn{4}{|l|}{ Uso de ATM } \\
\hline Sim & & 29 & 69 \\
\hline Não & & 13 & 31 \\
\hline Sexo & 29 & & \\
\hline Feminino & & 27 & 93 \\
\hline Masculino & & 2 & 7 \\
\hline Idade & 29 & & \\
\hline 60- 69 anos & & 6 & 20 \\
\hline 70- 79 anos & & 8 & 28 \\
\hline 80 anos e mais & & 15 & 52 \\
\hline Motivos de internação* /ATMs & 18 & & \\
\hline Doenças do aparelho respiratório & & 5 & 28 \\
\hline Lesão, envenenamento ou certas consequências de causas externas & & 5 & 28 \\
\hline Doenças do aparelho circulatório & & 4 & 22 \\
\hline Sinais, sintomas ou achados classificados em outra parte & & 4 & 22 \\
\hline Doenças endócrinas, nutricionais ou metabólicas & & 1 & 7 \\
\hline Outros & & 3 & 11 \\
\hline Não informado & & 11 & 61 \\
\hline Diagnósticos conclusivo* no uso de ATMs & 16 & & \\
\hline Doenças do aparelho respiratório & & 6 & 38 \\
\hline Doenças do aparelho circulatório & & 5 & 32 \\
\hline Doenças endócrinas, nutricionais e metabólicas & & 4 & 25 \\
\hline Doenças do aparelho digestivo & & 2 & 13 \\
\hline Outros & & 2 & 13 \\
\hline Doenças infecciosas e parasitárias & & 2 & 13 \\
\hline Lesão, envenenamento ou certas consequências de causas externas & & 1 & 6 \\
\hline Transtorno mentais e comportamentais & & 1 & 6 \\
\hline Neoplasias & & 1 & 6 \\
\hline Não informado & & 13 & 81 \\
\hline Período de internação**/ ATMs & 29 & & \\
\hline 7-14 dias & & 12 & 41 \\
\hline 15 a 21 dias & & 11 & 38 \\
\hline 22 ou mais dias & & 4 & 14 \\
\hline Quantidade de ATM/paciente & 29 & & \\
\hline Apenas 1 & & 12 & 41 \\
\hline Dois & & 15 & 52 \\
\hline 3 ou mais & & 2 & 7 \\
\hline \multicolumn{4}{|l|}{ Via de Administração dos ATMs } \\
\hline Via oral & 29 & 12 & 41 \\
\hline Intravenosa & & 23 & 79 \\
\hline Sem informação & & 1 & 3 \\
\hline \multicolumn{4}{|l|}{ Especialidade do Prescritor/ATMs } \\
\hline Clínico Geral & 29 & 28 & 97 \\
\hline Outros & & 1 & 3 \\
\hline \multicolumn{4}{|l|}{ Polifarmácia / ATMs } \\
\hline Sim & 29 & 29 & 100 \\
\hline Não & & 0 & - \\
\hline
\end{tabular}

(*) Classificação Internacional de Doenças (CID-11).

(**) Pacientes que continuaram internados (7\%; $n=2)$. Fonte: Autores (2020). 
No estudo observou-se o uso de 7 ATMs distintos, pertencentes a 7 classes constantes na Anatomical Therapeutic Chemistry (ATC), terceiro nível correspondente. Houve maior prevalência de uso das cefalosporinas de $3^{\mathrm{a}}$ geração, representadas pela ceftriaxona (59\%), seguida da classe das quinolonas representadas pelo levofloxacino (28\%) e macrolídeos, representado pela azitromicina (28\%), as demais classes de antibacterianos, suas respectivas dosagens e vias de administração estão descritas na Tabela 3.

Tabela 3 - Classificação dos ATMs conforme a Anatomical Therapeutic Chemistry (ATC), nível 3, de pacientes idosos internados no setor da clínica médica de um hospital público. Bahia, Brasil, 2018-2019.

\begin{tabular}{|c|c|c|c|c|}
\hline $\begin{array}{c}\text { Classe ATC } \\
\end{array}$ & Dose & $\begin{array}{c}\text { Via de } \\
\text { Administração }\end{array}$ & $\mathbf{N}$ & $\%$ \\
\hline $\begin{array}{c}\text { Cefalosporina de terceira geração: } \\
\text { (ceftriaxona) }\end{array}$ & $\begin{array}{l}500 \mathrm{mg} ; 1 \mathrm{~g} \\
12,5 \mathrm{mg} \text {. }\end{array}$ & $\begin{array}{l}\mathrm{VO} \\
\mathrm{IV}\end{array}$ & 17 & 59 \\
\hline $\begin{array}{c}\text { Quinolonas antibacterianos } \\
\text { (Levofloxacino) }\end{array}$ & $\begin{array}{l}5 \mathrm{mg} ; 6 \mathrm{mg} \\
500 \mathrm{mg}\end{array}$ & $\begin{array}{l}\text { VO } \\
\text { IV }\end{array}$ & 8 & 28 \\
\hline $\begin{array}{c}\text { Macrolídeos } \\
\text { (Azitromicina) }\end{array}$ & $\begin{array}{l}2 \mathrm{mg} \\
500 \mathrm{mg}\end{array}$ & VO & 8 & 28 \\
\hline $\begin{array}{c}\text { Penicilinas associadas ainibidores de beta-lactamase } \\
\text { (Piperacilina+ Tazobactam) }\end{array}$ & SI & SI & 1 & 3 \\
\hline $\begin{array}{c}\text { Quinolonas } \\
(\text { Ciprofloxacino })\end{array}$ & $500 \mathrm{mg}$ & $\begin{array}{l}\text { IV } \\
\text { VO }\end{array}$ & 4 & 14 \\
\hline $\begin{array}{c}\text { Cefalosporinas de quarta geração } \\
\text { (Cefepime) }\end{array}$ & $1 \mathrm{~g}$ & VO & 1 & 3 \\
\hline $\begin{array}{l}\text { Lincosamidas } \\
\text { (Clindamicina) }\end{array}$ & $\begin{array}{l}100 \mathrm{ui} \\
150 \mathrm{mg} \\
300 \mathrm{mg}\end{array}$ & $\begin{array}{l}\text { IV } \\
\text { VO }\end{array}$ & 7 & 24 \\
\hline $\begin{array}{l}\text { Derivados de Imidazol } \\
\text { (Metronidazol) }\end{array}$ & $\begin{array}{l}5 \mathrm{mg} / \mathrm{ml} \\
5 \mathrm{mg} / \mathrm{ml}\end{array}$ & $\begin{array}{l}\text { IV } \\
\text { IV }\end{array}$ & 2 & 7 \\
\hline
\end{tabular}

SI: Sem informação; VO: via oral; IV: Intravenosa. Fonte: Autores (2020).

\section{Discussão}

Os principais achados do presente estudo apontam uma alta prevalência (69\%) do uso de ATMs na população idosa analisada. Foi possível identificar os grupos de medicamentos mais utilizados, bem como evidenciar a alta prevalência de ATMs por estes idosos, estando em consonância com outros estudos nacionais e internacionais.

Em cenário nacional, um estudo em dezoito hospitais brasileiros adotados pelo projeto internacional Point Prevalence Global Survey of Antimicrobial Consumption and Resistance (GLOBAL-PPS) mostrou que a prevalência do consumo de ATMs nas cincos macrorregiões para pacientes com idade média de 58 anos foram 48,6\% para o Sul e 60,4\% para o Nordeste (Porto et al., 2020). Em contrapartida, outro estudo realizado em hospitais europeus mostrou que a prevalência para o consumo foi menor, (30\%) (ECDC, 2013). Ao contrapor os achados destes estudos, é evidente que taxa do consumo de ATMs no Brasil é excessiva quando comparado a países europeus, esse predomínio pode ser explicado devido à escassez de medidas e ações educativas no País e de exames laboratoriais, bem como ausência de restrição do uso hospitalar.

No que se refere ao uso dos ATMs, a predominância do sexo para o uso desses medicamentos nesta faixa etária foi para o sexo feminino com (93\%). Em consonância com o achado da literatura, as mulheres procuram mais os serviços de saúde, além de maiores contatos de apoio social e, sobretudo, cuidados regulares do dia a dia (Levorato et al., 2014).

Comparativamente, um realizado em um hospital universitário na cidade de Belém do Pará mostrou um equilíbrio entre os sexos de forma discreta, sendo possível comprovar a predominância para o sexo masculino foi de $56,5 \%$ (Sousa \& 
Silva, 2015), enquanto que no hospital do Mato Grosso do Sul, o predomínio de idosos internados constituiu-se para sexo masculino 58,2\% (Souza et al., 2017).

Em concordância com estes achados, um estudo internacional conduzido em um hospital na Itália, os pesquisadores mostraram que os percentuais para o uso de ATMs em idosos foi semelhante entre homens 48,3\% e mulheres 48,0\% (Ardoino et al., 2019). Entretanto, é importante destacar que estes achados podem ser divergentes e explicados devido às consequências das demandas encontradas durante o período e regionalidade de cada estudo.

Em relação a idade, os resultados mostraram que os pacientes com igual ou superior a 80 anos foram mais prevalentes 52\%. Todavia, estudos semelhantes realizados por Colet \& Neves (2015), no hospital da Unidade de Terapia Intensiva (UTI) no Rio Grande do Sul- RS, os pacientes possuíam entre 60 a 80 anos, o que representou 48\% com uso de ATMs.

Sendo assim, esse achado é justificado justamente porque os pacientes longevos estão mais propícios as alterações fisiológicas e comprometimento do sistema imunológico, além de precisarem de cuidados mais específicos e realizações de procedimentos invasivos (Garcia et al., 2013).

No que concerne aos motivos de internações e diagnósticos, estudo do tipo descritivo baseado no Sistema de Informações Hospitalares do Sistema Único de Saúde (SUS), os pesquisadores avaliaram as causas comuns de internações de idosos no Brasil, e observaram que nas diferentes regiões brasileiras as causas foram principalmente para as doenças do aparelho circulatório e aparelho respiratório (Barbosa et al., 2019).

Neste estudo, identificou que os ingressos de internação foram maiores quando classificados por "Sinais, sintomas não classificados em outra parte", sendo 43\% dos pacientes. Apesar de não encontrar na literatura dados que justifiquem este resultado, acredita-se que por englobar uma quantidade maior de sintomas, referentes as doenças do aparelho respiratório, circulatório, sistema nervoso, além dos que afetam o cognitivo, estado emocional e comportamental, entre outros, levam a uma maior recorrência de pacientes internados acometidos por essas doenças (Datasus, 2020).

Em contrapartida, observou-se que os motivos para o uso de ATMs durante o estudo, foram maiores para as doenças do aparelho respiratório (28\%), seguidas de "Lesões, envenenamento e algumas outras consequências de causas externas" (28\%), estes dados corroboram com um estudo realizado no hospital do Canadá em que houve predominância dos mesmos motivos (Latham et al., 2014). No que remete as lesões, os achados da literatura mostram que esses fatores podem estar relacionados com acidentes domésticos, pois são fatores propensos aos idosos, bem como a violência urbana (Teixeira et al., 2017).

Já em relação as doenças que acometem o aparelho circulatório, a predominância para o uso de ATMs foi de 32\%, uma prevalência discreta quando comparada a um estudo realizado no hospital de Belo Horizonte-MG com idosos (35,5\%) (Cruz et al., 2020).

Cabe destacar que o resultado encontrado no presente estudo pode estar relacionado, dentre outros fatores, a um menor número de indivíduos internados. Ressalta-se que as doenças crônicas que contribuem para esses diagnósticos nos idosos são os problemas cardiovasculares, envolvendo o infarto agudo do miocárdio (IAM), hipertensão arterial sistêmica (HAS), insuficiência cardíaca e diabetes, dentre outros inclusos da síndrome metabólica, contudo, apesar dessas doenças não serem transmissíveis, afetam com maior constância os idosos (Teixeira et al., 2017).

No que concerne as doenças que acometem o aparelho respiratório, um dos principais diagnósticos é a pneumonia que afeta as vias aéreas inferiores, principalmente dos idosos (Souza \& Silva, 2015). Este dado está em concordância com um estudo realizado no hospital da Inglaterra, em que foram avaliadas todas as hospitalizações e constataram que os pacientes acima de 60 anos são mais propensos as causas de pneumonia e doenças do trato respiratório inferior (ITR) (Millett et al., 2013). Vale ressaltar que estes indivíduos já estão debilitados e com o sistema imunológico afetado, ou seja, estão propícios a adquirir essas infecções, além de ser explicados pela presença de comorbidades devido a doenças associadas a idade. 
É importante destacar que os Beta-lactâmicos foi a classe mais prescrita durante a internação hospitalar para essas doenças, sendo as cefalosporinas de terceira geração, particularmente, a ceftriaxona a mais prevalente (59\%). Além disso, a classe dos quinolonas antibacterianos e dos macrolídeos, respectivamente levofloxacino e azitromicina também apresentaram destaques (28\%). Vale ressaltar que a ceftriaxona é amplamente utilizado em problemas respiratórios e infecções de tecidos moles tendo como ação de amplo espectro contra as bactérias gram negativas (Silva et al., 2014) bem como o levofloxacino e azitromicina que também são medicamentos utilizados em problemas respiratórios e infecções do trato urinário, além disso, promove melhores farmacocinéticas e menos efeitos adversos (Selbach et al., 2017).

Um estudo realizado em Centros de Saúde em Portugal, avaliou o uso de ATMs em todas as faixas etárias e constatou que para as doenças do aparelho respiratório e do trato urinário, as penicilinas representaram 43,6\% dos medicamentos mais utilizados nos indivíduos mais velhos, em seguida as cefalosporinas de terceira geração ceftriaxona $(26,8 \%)$ (Ramalhinho et al., 2015).

Em contrapartida, um estudo transversal realizado no Maranhão, no setor de terapia intensiva com pacientes acima de 60 anos, verificou que dentro das classes das cefalosporinas a Cefepime foi o medicamento mais utilizado (Alves et al., 2019). Entretanto, esse medicamento é mais prescrito para infecções do trato urinário, todavia, no presente estudo não houve um diagnóstico conclusivo para essa condição clínica.

Quanto ao tempo de internação a maioria dos pacientes permaneceram internados de 7 a 14 dias (41\%) com a média de 15 dias durante o período de acompanhamento deste estudo. Estes dados foram similares com o estudo de Rodrigues e Bertold (Rodrigues \& Bertoldi, 2010), em que a média do tempo de internação com o uso de ATMs foi 14 dias. No entanto, no estudo realizado Silva et al. (2017), conduzido no hospital público do Pará, a média do tempo de internação foi de 7 dias. O dado do presente estudo houve semelhança com estudo internacional no hospital na Alemanha, em que a média para o tempo de permanência foi de 9 dias (Beier et al., 2019).

Cabe destacar que a oscilação quanto as médias podem estar relacionadas a patologia do paciente, às especialidades médicas que cada hospital disponibiliza, bem como aos tratamentos efetuados durante o tempo de permanência. Por outro lado, há também uma preocupação durante esses períodos de internações, já que os pacientes mais velhos tendem a ficar mais expostos a diversas infecções, risco de erros de medicação e eventos adversos (Colet et al., 2011).

Em relação à média do uso de ATMs por paciente, o resultado do estudo $(1,6)$ obteve uma semelhança quando comparado a outro estudo em um hospital de Belo Horizonte-MG em que a média para o uso de ATMs em idosos foi 2 (Cruz et al., 2020). Este dado também se aproxima do resultado de um estudo realizado em hospitais na República Eslovaca, em qu média foi de 1,2 ATMs por pacientes idosos (Bražinová, 2016).

Vale salientar que a terapia empírica na maioria das vezes potencializa uma ação terapêutica de amplo espectro. Sendo assim, o tratamento realizado nessa modalidade é importante no processo de praticidade do atendimento para o paciente, embora os exames laboratoriais sejam imprescindíveis para um resultado coerente com a doença do paciente e seguimento de uma terapia antimicrobiana mais específica ao agente etiológico (Santos et al., 2016; Costa \& Júnior, 2017).

Quanto a via de administração observa-se uma maior frequência para os medicamentos administrados por via intravenosa, uma vez que os pacientes já estão debilitados e precisariam de um efeito farmacológico imediato, além de permitir controle da dose, pois os pacientes longevos possuem dificuldade de deglutição (Colet et al., 2011).

No que diz respeito a polifarmácia, é comum que tenha alta prevalência na população idosa, conforme foi observado neste estudo. Esta prática torna-se os idosos mais expostos aos riscos da farmacoterapia, pois eles são mais suscetíveis a utilização de vários medicamentos decorrentes das doenças crônicas e alterações fisiológicas do envelhecimento. Além disso, contribui na baixa adesão ao tratamento, visto que esses fatores cooperam para os erros de medicações e interações 
medicamentosas, já que estes erros com uso de ATMs podem proporcionar uma resposta terapêutica ineficaz que pode estar relacionada com a morbidade e mortalidade, além de aumento dos custos aos sistemas de saúde (Rodrigues \& Oliveira, 2016).

Em todos os prontuários analisados neste estudo, observou-se a ausência da duração do tratamento, a omissão desta informação principalmente para o uso dos ATMs pode comprometer à saúde do paciente, uma vez que essa variável é indispensável na prescrição afim de evitar um uso contínuo desnecessário ou a falta da instituição de uma terapia necessária, além de ser um fator para possíveis presenças de cepas resistentes (Silva et al., 2017). O controle do uso de ATMs de acordo com Antimicrobial Stewardship Program (ASP) é fundamental para melhoria terapêutica associadas a dose, via de administração e duração do tratamento (Anvisa, 2020).

A informação do peso também esteve ausente nos prontuários, a falta desses elementos caracteriza-se como erros, pois os pacientes internados, principalmente os idosos estão submetidos a diversas alterações e manifestações clínicas resultantes do envelhecimento, por isso, a observação do peso deve ser avaliada para minimizar a ocorrência de superdosagem ou subdosagem do medicamento (Mota et al., 2018). No que se refere a dose, comparada com as informações da bula não houve divergências (Anvisa, 2020) porém houve omissão de 3\% de um dos medicamentos. Esse dado se aproxima com o estudo de Castro et al. (2019), em que houve 6\% da ausência da dose.

Frente os problemas identificados associados a essa prática neste estudo, o Programa Nacional de Segurança do Paciente (PNSP) estabelecido pela portaria $n^{\circ} 529$ de $1^{\circ}$ de Maio de 2013 e instituído pelo Ministério de Saúde tem como propósito aprimorar a qualidade da assistência nos hospitais, além disso, promover a segurança dos pacientes e tomar medidas para padronização dos protocolos, afim de avaliar as prescrições de ATMs e indicações, e suas respectivas informações antes do uso (Anvisa, 2020; Brasil, 2019).

Portanto, é importante ressaltar que tais problemas citados podem ser resolvidos com a presença de um farmacêutico clínico, pois este profissional contribui no cuidado para acompanhamento das prescrições que deve ser elaborada completa junto a equipe multiprofissional, pois eles são profissionais indispensáveis para que seja prestada a melhor segurança e assistência ao paciente, principalmente os idosos que necessitam de atenção mais específica (Carneiro et al., 2019). Além disso, o compartilhamento das informações deve ser clinicamente pertinente, pois estes assuntos são consideráveis importantes entre os profissionais de saúde para proporcionar uma ação farmacoterapêutica no âmbito hospitalar.

Diante das discussões abordadas neste estudo, identificamos algumas limitações no que se refere a possibilidade de omissão dos dados nos prontuários, o que impediu fazermos inferências mais profundas sobre a adequação do uso dos ATMs nesta população. Além disso, não foram verificados os exames laboratoriais a fim de acompanhar e comparar os dados da terapia medicamentosa. É importante frisar também que esta pesquisa foi realizada em um único hospital e que esses dados não podem ser generalizados, dado a consideração de fatores internos que podem afetar o perfil da prescrição de ATMs em cada hospital.

Contudo, através desses achados podemos listar que o uso de ATMs sem critérios rigidamente definidos pode facilitar a ocorrência da resistência bacteriana, uma vez que padronizar o uso de ATMs no ambiente hospitalar é essencial, já que essa classe é uma das mais utilizadas, além de implementar programas para o seu uso racional através da Comissão de Controle e Infecção Hospitalar (CCIH) para avaliar padrões do seu consumo.

Os dados deste estudo destacam também, a necessidade do diagnóstico conclusivo da patologia, para que o paciente obtenha um tratamento eficiente e adequado. Através destas questões, este estudo pode aumentar o número de pesquisas relacionadas a esse tema, trazer novas informações sobre a temática, incentivar o que consequentemente pode influenciar em futuras pesquisas. Ademais, destaca-se a importância do acompanhamento de um farmacêutico clínico neste setor, para avaliar as prescrições e otimizar uma terapêutica efetiva e segura, especialmente em virtude dos erros que foram observados, no que compete aos fármacos. 


\section{Conclusão}

Pôde-se concluir que o uso de ATM neste estudo obteve uma alta prevalência (69\%), sendo mais predominante no sexo feminino e em idosos com idade acima de 80 anos. As prescrições foram mais frequentes para as doenças do aparelho respiratório e circulatório, sendo que os fármacos mais utilizados foram a ceftriaxona, levofloxacino e azitromicina.

A partir destes resultados, observa-se a necessidade em conhecer o uso dos ATMs nos hospitais, principalmente na geriatria, para subsidiar a elaboração de protocolos clínicos, reduzir o tratamento por terapia empírica e realizar exames laboratoriais com o intuito de identificar os microrganismos, além de ser importante a interação da equipe multiprofissional para evitar o aumento da resistência bacteriana e impedir que possíveis erros quanto as prescrições ocorram. A partir disso, propõe-se que estudos futuros considerem a avaliação de parâmetros clínicos e laboratoriais, bem como informações sobre intervenções farmacêuticas, a fim de direcionar ações específicas relacionadas ao uso racional da terapia antimicrobiana.

\section{Referências}

Alves, F., Júnior, L. Matias, E. Vieira, S. Santos, B. \& Dias, T. (2019). Perfil das prescrições de antibioticoterapia em uma unidade de terapia intensiva adulto de um hospital de referência cirúrgica no estado do Maranhão Profile of antibiotics prescription in an adult intensive care unit at a surgical reference hospital in th. Revista Eletrônica Acerco Saúde. 34 (1):1-7.

ANVISA: Agência Nacional de Vigilância Santiária. Consultas. https://consultas.anvisa.gov.br/.

Ardoino, I., Mannucci, P.M., Nobili, A., Tettamanti, M. Pasina., \& L. Corrao, S. (2019). Uso de antibióticos e fatores associados em uma grande amostra de idosos hospitalizados. J Global Antimicrob Resist. 1 (19):167-172.

Baclet, A. N., Ficheur, G. Alfandari, S. Ferret L. \& Chazard, E, Beuscart, J. (2017). Explicit definitions of potentially inappropriate prescriptions of antibiotics in older patients : a compilation derived from a systematic review. Int J Antimicrob Agents. 50 (5): 1-35.

Barbosa, A. T. F., Carneiro, J. A. 1., Ramos, G. C. F., Leite, M. T. \& Caldeira, A. P. (2019). Fatores associados à Doença Pulmonar Obstrutiva Crônica em idosos. Ciência \& Saúde Coletiva. 22 (1): 63-73.

Beckett, C.L., Harbarth, S., \& Huttner, B. Special considerations of antibiotic prescription in the geriatric population. (2014). CMI. 21 (1): 1-7.

Beier, D., Weiß, C. Hagmann, M., Balaban, Ü. \& Thiel, M. Schneider-lindner, V. (2019). Is antibacterial treatment intensity lower in elderly patients? A retrospective cohort study in a German surgical intensive care unit. BMC Health Services Research. 19 (1): 1-9.

Bertol, C. D., Anzolin, A. P., Silva, L. H., Dalbosco, A. K., Portella, M. R. \& Hahn, S. R. (2020). Avaliação das infecções hospitalares em idosos. Revista Interdisciplinar de Estudos em Saúde. 9 (1): 1-19.

Brasil. Antimicrobianos: erros de medicação, riscos e práticas seguras na sua utilização. (2019) ISMP. (7): 2-14.

Brasil. Ministério da Saúde. Conselho Nacional de Saúde. Resolução no 466, de 12 de dezembro de 2012. (2013). Dispõe sobre diretrizes e normas regulamentadoras de pesquisas envolvendo seres humanos. Diário Oficial República Federativa do Brasil, Brasília, DF. https://bvsms.saude.gov.br/bvs/saudelegis/cns/2013/res0466_12_12_2012.html.

Carneiro, L. F., Khouri A. G., Santos, S. O., \& Silveira A. C. S. (2019). Atribuição Do Farmacêutico Na Comissão De Infecção Hospitalar Quanto ao Uso de Antimicrobianos. Rev Ref em Saúde da Fac Estácio Sá Goiás. 2 (3):69-74.

Castro, A. F., Oliveira, J. P. \& Rodrigues, M. C. S. (2019). Erro de administração de medicamentos anti-infeciosos por omissão de doses. Acta Paul Enferm. 32 (6): 667-73.

Cazarim, M. S., \& Araújo, A. L.A. (2011). O paciente idoso sob o aspecto da utilização de antimicrobianos : repercussão ao sistema público de saúde brasileiro. Revista de Ciências Farmacêuticas. 32 (3): 305-11.

Colet, C., \& Wazlawick., M. K. M. (2011). Perfil de uso de antimicrobianos por idosos em hospital de nível IV do RS em 2010. Context Saúde; 11 (20): 11971202 .

Costa, A. L. P., \& Junior, A.C.S.S. (2017). Resistência bacteriana aos antibióticos e Saúde Pública : uma breve revisão de literatura. Estação Científica. 7 (1): 45-57.

Cruz, L. F., Marçal, A. G., Reis, A. M., \& Bertollo, C. M. (2020). Terapia antimicrobiana em idosos: perfil de uso e avaliação da qualidade da prescrição. Revista Brasileira de Farmácia Hospitalar e Serviços de Saúde. 11(1):1-8.

Datasus. Sintomas, sinais e achados anormais de exames cínicos e do laboratório, não classificados em outra parte. http://www2.datasus.gov.br/cid10/V2008/WebHelp/r00_r99.htm.

Dylis, A., Boureau, A.S., Coutant, A., Batard, E. Javaudin, F. \& Berrut, G. (2019). Antibiotics prescription and guidelines adherence in elderly: impact of the comorbidities. BMC Geriatrics. 19: (291) 1-6. 
ECDC. (2013). Point prevalence survey of healthcare-associated infections and antimicrobial use in European acute care hospitals. 5 (3): 1-83.

Flores, T.G., Costa., G.S., Oliveira, R. Pedro, F. L., Cruz, I.B.M., \& Lampert, M.A. (2019). Prescrição de antimicrobianos para idosos hospitalizados : análise do benefício e associação com implementação de limitação de esforço terapêutico e cuidados paliativos. Journal of Epidemiology and Infection Control. 9 (4):1-7.

Garcia, L. M., Oliveira, C. Braga., C.A., Aurea, G. Damasceno A, \& Mota É.C. (2013). Perfil epidemiológico das infecções hospitalares por bactérias multidrogarresistentes em um hospital do norte de Minas Gerais. Revista de Epidemiologia e Controle de Infecção. 3 (2):45-49.

Giarratano, A., Green S. E. L., \& Nicolau, D.P.(2018). Review of antimicrobial use and considerations in the elderly population. Clinical Interventions in Aging. 13 (1): 657-667.

Gleeson, L., Dalton, K. Mahony, D. O., \& Byrne, S. A (2020). Systematic Review and Narrative Synthesis. Res Soc Adm Pharm. 16 (8): $1017-1025$.

Goulart, L. S., Carvalho, A. C., Lima, J.C., Pedrosa, J. M., Lemos, \& P. L., Oliveira, R. B. (2014). Consumo de medicamentos por idosos de uma Unidade Básica de Saúde de Rondonópolis/MT. Estudo Interdisciplinar Envelhecimento. 19 (1): 79-84.

Izaias, É. M., Rossaneis, M. Â. \& Belei, R. A. (2014). Custo e caracterização de infecção hospitalar em idosos. Ciência \& Saúde Coletiva. 19 (8): $3395-402$.

Latham, L. P., \& Ackroyd-stolarz, S. Emergency Department Utilization by Older Adults : a Descriptive Study. (2014). Can Geriatr. 17 (4):118-125.

Levorato, C. D., \& Nunes, A. A. (2014). Fatores associados à procura por serviços de saúde numa perspectiva relacional de gênero Factors associated with the demand for health services from a gender-relational perspective. Ciência \& Saúde Coletiva. 19 (4): $1263-1274$.

Microsoft Office. Excel (versão 15.0). [Software Computer]. Office; 2013.

Millett, E. R. C., Quint, J. K., Smeeth, L., Daniel, R. M., \& Thomas, S. L. (2013). Incidence of Community-Acquired Lower Respiratory Tract Infections and Pneumonia among Older Adults in the United Kingdom : A Population-Based Study. Plos One: 8 (9):1-11.

Mota, I. V. R., Almeida, P. H. \& Lemos., L. B. (2018). Prescription errors and administration of injectable antimicrobials in a public hospital. Revista Brasileira Farmácia Hospitalar Serviços Saúde. 9 (1): 2-7.

Msslvmzk, A. B. (2016). Point prevalence study of antimicrobial usage in acute care hospitals in the Slovak Republic. ECDC. 93 (4):403-409.

Neves, C., \& Colet, C. (2015). Perfil de uso de antimicrobianos e suas interações medicamentosas em uma uti adulto do Rio Grande do Sul. Revista de Epidemiologia e Controle de Infecção.5(2): 65-71.

Pereira A. S. et al. (2018). Metodologia da pesquisa científica. [free e-book]. Santa Maria/RS. Ed. UAB/NTE/UFSM.

Porto, A. P. M., Goosens, H. Verspoten, A. \& Costa, S. F. (2020). Global Point Prevalence Survey (Global-PPS) of Antimicrobial Consumption in Brazilian Hospitals. J Hosp Infect. 104 (2): 165-171.

Ramalhinho, I. Gomes., L. F. Filipe, C., Cavaco, A., \& Cabrita, J. ( 2015). Padrão de prescrição de antibióticos no Algarve: características do doente e dispersão da terapêutica. Rev Port Saude Publica. 33 (2): 207-21.

Rodrigues, F. A., \& Bertoldi, A. D. (2010). Perfil da utilização de antimicrobianos em um hospital privado The profile of antimicrobial utilization in a private hospital. Ciência \& Saúde Coletiva. 14 (1):1239-1247.

Rodrigues, M. C. S., \& D. O. C. (2016). Interações medicamentosas e reações adversas a medicamentos em polifarmácia em idosos: uma revisão integrativa. Revista Latino Americano de Enfermagem. 24 (1): 1-17.

Santos, R. G., Alves., C. D. S., Lemos, L. B., Jesus, I. S. \& Lemos, G. S. (2016). Prescrições de antimicrobianos de uso restrito de pacientes internados em um hospital e ensino. Rev Bras Farm Hosp e Serviços Saúde. 7(1):8-12.

Secretaria Estadual de Saúde da Bahia (SESAB). Hospital Geral Prado Valadares (HGPV) .Salvador, [s/a]. <http://www.saude.ba.gov.br/hospital/hospitalgeral-prado-valadares/>.

Selbach, C. V., Blanco, B. S., Mello, L. D., \& Cassão, G. (2017). Prevalência do uso do antibiótico levofloxacino em hospital de bagé-rs no mês de agosto de 2016. Congrega Urcamp. 1-2.

Silva, A. G., \& Garbaccio, J. L. (2016). Registro do uso de antimicrobianos em Instituições de Longa Permanência para Idosos. Revista Brasileira Geriatria Gerontologia. 19 (2): 325-334.

Silva, J, S. D., Almeida, P. H. R. F., Perini, E. Pádua., C. A. M. \& Rosa, M. B. (2017). Erros da Prescrição e Administração envolvendo um medicamento potencialmente perigoso. Revista de Enfermagem. 11(10):3707-17.

Silva, T. F. A., Filho, M. A. A., Brito, M. R. M B., \& Freitas, R. M. (2014). Mecanismo de ação, efeitos farmacológicos e reações adversas da ceftriaxona: uma revisão de literatura. Revista Eletrônica de Farmácia. 11 (3):48-57.

Sousa, K .C., \& Silva, M. V. (2015). Tendências de prescrição de antimicrobianos em idosos hospitalizados em um hospital universitário. Revista Saúde e Pesquisa. 8 (3): 501-508.

Souza, F., Baroni, M. R. F. (2017). Perfil de utilização de antimicrobianos na unidade de terapia intensiva de um hospital público. Rev Bras Farmácia Hosp e Serviços Saúde.8 (4):37-44. 
Research, Society and Development, v. 10, n. 14, e558101420683, 2021

(CC BY 4.0) | ISSN 2525-3409 | DOI: http://dx.doi.org/10.33448/rsd-v10i14.20683

Statistical Package for the Social Sciences (SPSS). (2012). IBM SPSS Statistics (versão 21). <https://www.ibm.com/br-pt/analytics/spss-statistics-software>.

Tavares, C.A., Veras, M.C.B., Carla, A. Araújo, S.E.S., \& Lima, M.D.F.S. (2015). Avaliação da prescrição de antimicrobianos para infecção relacionada à assistência à saúde em um Hospital Escola de Recife - PE Evaluation of antimicrobial prescribing infection related to health care in. Revista de Epidemiologia e Controle de Infecção. 5 (3):123-30.

Teixeira, J.J.M., Bastos, G.C.F.C., \& Souza, A.C.L. (2017). Perfil de internação de idosos Profile of Hospitalization of the elderly. Rev Soc Bras Clin Medica. (62):15-20.

WHO. (2002). Active Ageing - A Police Framework. A Contribution of the World Health Organization to the second United Nations World Assembly on Aging. World Health Organization (WHO) <https://extranet.who.int/agefriendlyworld/wp-content/uploads/2014/06/WHO-Active-Ageing-Framework.pdf>.

WHO. (2018). World Health Organization. Estrutura e Princípios ATC/DDD. Instituto Norueguês de Saúde Pública, Noruega https://www.whocc.no/atc/structure_and_principles/.

WHO. World Health Organization CID-11: classificação estatística internacional de doenças e problemas relacionados à saúde. 